\title{
Endodontic Management of Dental Infection Mimicking Skin Lesion
}

\author{
Saloni Gattani ${ }^{1}$, Deepak Raisingani², Prachi Mital ${ }^{3}$, Lalita Poonia ${ }^{4}$, Aarushi Chopra ${ }^{5}$ \\ ${ }^{1}$ Department of Conservative Dentistry \& Endodontics, Mahatma Gandhi Dental College \& Hospital, \\ Jaipur, Rajasthan, India.
}

\section{INTRODUCTION}

Medical literature has well documented cases of cutaneous sinus tracts of odontogenic origin. However, such lesions still continue to present with a diagnostic problem to the clinician. Several cases that have been reported have revealed that patients have undergone multiple treatment modalities like surgical excisions, radiotherapies, biopsies and repeated antibiotic regimens, all of which have eventually failed, with recurrence of the cutaneous sinus tract, as the primary aetiology reported to dental origin was mostly misdiagnosed or overlooked. This case report presents the treatment of an extraoral draining sinus by the means of conventional nonsurgical root canal therapy.

An endodontic emergency develops when patient experiences severe pain or an intra / extra oral swelling. Often times, the patient immediately reports to a dentist. However, at times, the patient does not seek any dental treatment and later may report with an extra oral cutaneous lesion. One of the reasons for an extra oral sinus of odontogenic origin is chronic inflammation of pulpal origin. ${ }^{1}$ This cutaneous lesion presents as a pathway traversing the alveolar bone, that begins either at the apex of an infected tooth or an infected segment of dental alveolus. This tract drains the infected material i.e., the abscess through the skin to the external surface. Site of the opening of sinus tract is dependent of the location of cortical plate perforation by the inflammation process and its relationship to the facial-muscle attachments. The infection can spread to extra-oral regions in cases when the apices of the teeth are located either above the maxillary muscle attachments or below the mandibular muscle attachments. They are more common in infected mandibular teeth $(80 \%)$ than the maxillary teeth $(20 \%){ }^{2}$

An extraoral sinus tract of odontogenic origin is often times confused with diseases including skin infections, occluded sweat gland duct, osteomyelitis, neoplasms, tuberculosis, actinomycosis, and congenital midline sinus of the upper lip. ${ }^{3} \mathrm{~A}$ thorough clinical and radiographic evaluation must be emphasised upon to rule out any possible aetiological factor.

This case report elaborates a case of patient presenting with an extra oral sinus secondary to chronic periapical infection.

\section{PRESENTATION OF CASE}

A 23-year-old young female patient reported to the Department of Conservative dentistry \& Endodontics with the chief complaint of pain in lower right back tooth region since 20 days. Extra oral examination revealed a draining sinus in the submandibular region. The lesion was erythematous, non-tender and slightly stiff about $1 \mathrm{~cm}$ in diameter. (Figure 1) Intraoral examination revealed a deep carious right mandibular second molar (\#47) which was tender to percussion with grade 1 mobility. (Figure 1)
Corresponding Author: Dr. Saloni Gattani,

B-41, Devnagar, Tonk Road, opposite Kamal \& Company, Jaipur- 302018, Rajasthan, India. E-mail: salonigattani@gmail.com

DOI: $10.14260 / j e m d s / 2020 / 837$

How to Cite This Article:

Gattani S, Raisingani D, Mital P, et al. Endodontic management of dental infection mimicking skin lesion. J Evolution Med Dent Sci 2020;9(50):3819-3822, DOI: 10.14260/jemds/2020/837

Submission 26-08-2020,

Peer Review 26-10-2020,

Acceptance 03-11-2020,

Published 14-12-2020.

Copyright (C) 2020 Saloni Gattani et al. This is an open access article distributed under Creative Commons Attribution License [Attribution 4.0 International (CC BY 4.0)] 
Radiographic examination revealed deep caries involving pulp with large periapical radiolucency in relation to (irt) 47 . The patient was further advised for a CBCT (Cone Beam Computed Tomography) scan to know the extent of the periapical lesion and cortical bone perforation. The CBCT scan clearly demonstrated a well-defined periapical radiolucency, perforating the buccal cortical plate with an approximation of root apices with the mandibular canal. (Figure 2). Based on the clinical and radiographic examinations, a diagnosis of pulpal necrosis with chronic periapical abscess with cutaneous sinus tract irt 47 was formulated.

\section{DISCUSSION OF MANAGEMENT}

Nonsurgical endodontic therapy was planned. Endodontic therapy was initiated. Tooth was anaesthetised with $2 \%$ lidocaine with 1:200,000 epinephrine under rubber dam isolation. Access opening was made with Endoaccess bur (Dentsply Maillefer). Orifices were located with the help of DG16 endodontic explorer (Hu-Friedy, Chicago, USA). Working length was established with $10 \mathrm{~K}$ file (Dentsply Maillefer, Switzerland) (Figure 3).
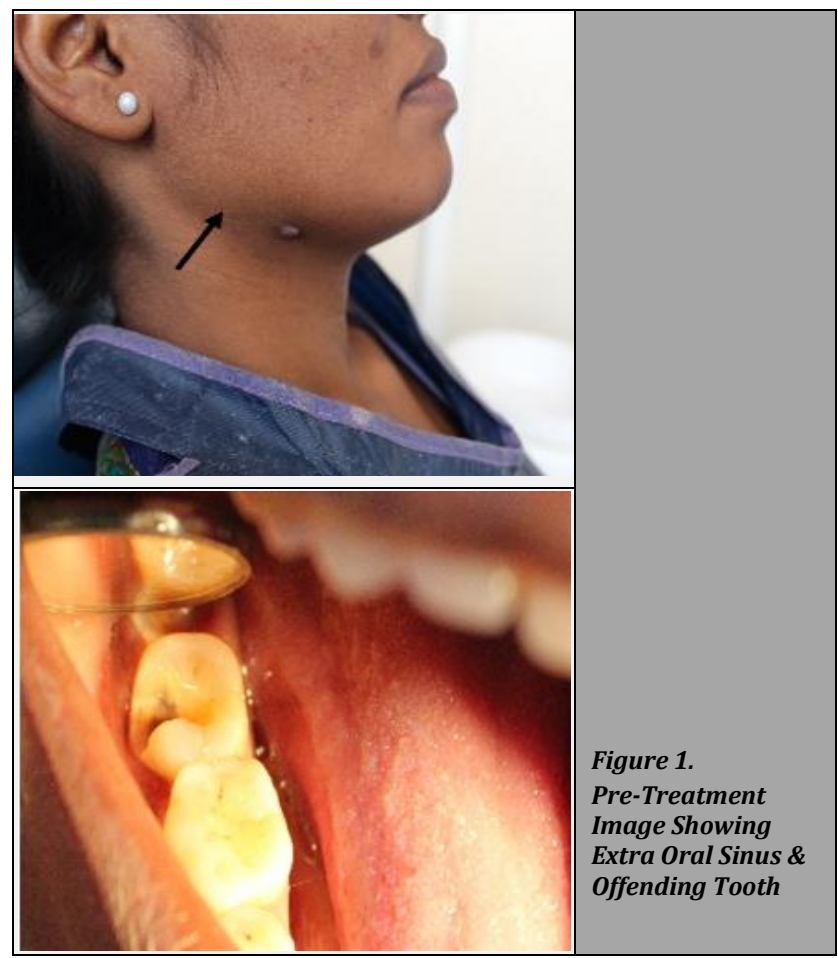

The canals were cleaned and shaped on the same visit up to $25,4 \%$ in mesiobuccal and mesiolingual canals and 20,6\% in distal canal by Hyflex CM rotary files (Coltene \Whaledent) using $15 \%$ EDTA (Ethylenediaminetetraacetic acid) (Glyde, Dentsply Maillefer) with copious irrigation with saline and 2 $\%$ chlorhexidine (CHX Plus). $\mathrm{NaOCl}$ (Sodium hypochlorite) was not used for irrigation due to continuous pus drainage from root canal. Post the biomechanical preparation, calcium hydroxide powder (Prevest DentPro) mixed with chlorhexidine was used as an intracanal dressing for 2 weeks. The sinus tract was irrigated and debrided with Povidoneiodine and normal saline. Patient was recalled after 2 weeks and showed healing of the sinus opening with no clinical signs and symptoms.
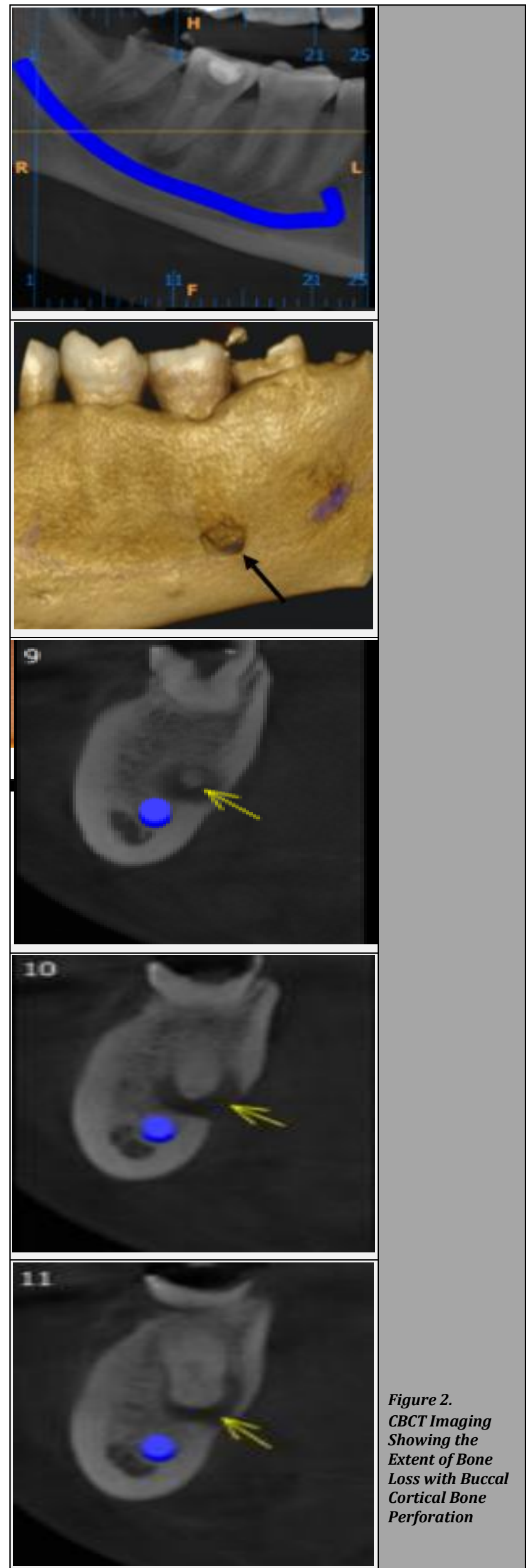

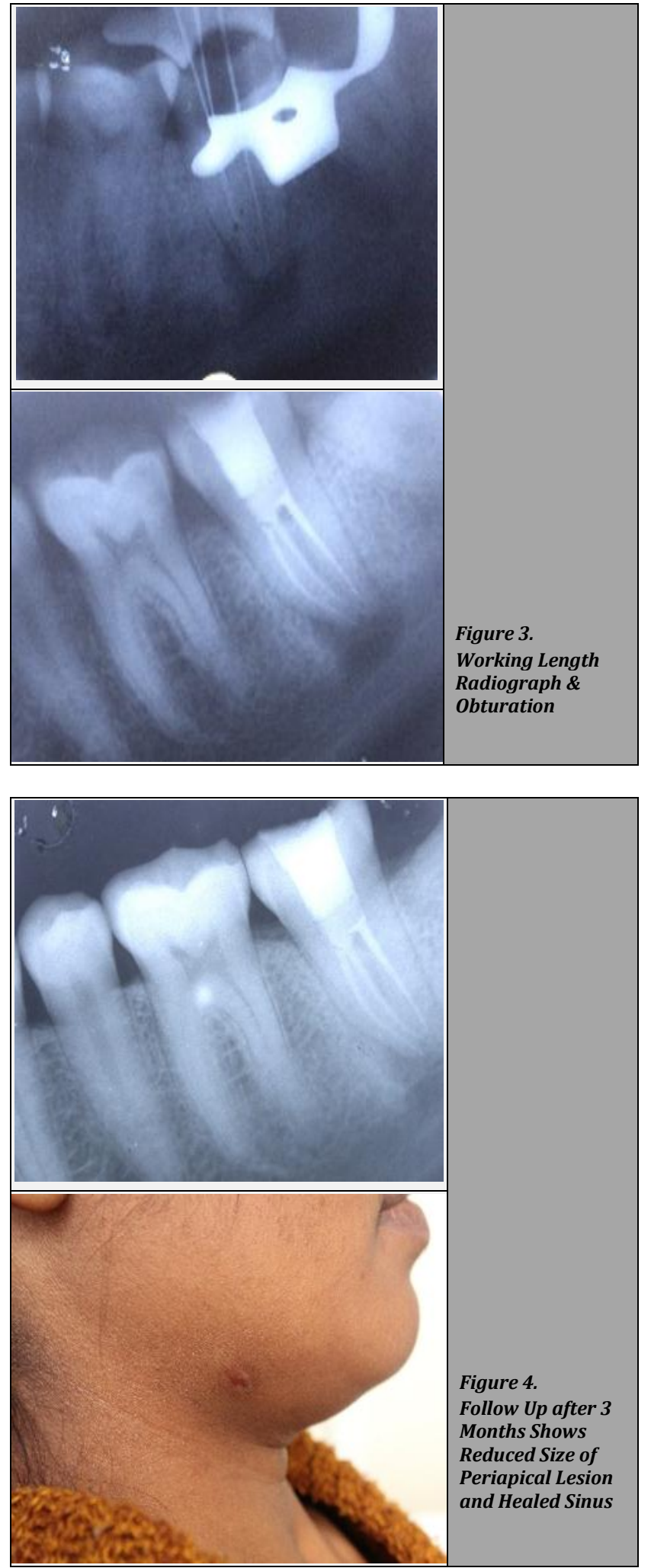

The dressing was removed from the canals with copious irrigation with $5.25 \% \mathrm{NaOCl}$ and saline. Canals were dried and obturation was done followed by composite resin restoration to prevent any microleakage (Figure 3). Patient was called for follow up after 3 months and showed healed sinus (Figure 4).

\section{DISCUSSION}

Dental pathosis is one of the most common cause of cutaneous sinus tracts occurring on the face and neck region and should always be the primary suspect in the differential diagnosis. The majority of dental sinus tracts develop within the oral cavity. Whenever an extraoral sinus tract occurs, it mostly develops in close proximity to the offending tooth. ${ }^{4}$

Acute periapical abscess drains along the path of least resistance via an intraoral or extraoral opening in the form of a sinus tract or may spread to deeper tissues leading to fascial space infection. Opening of the sinus tract depends on the path the inflammatory process follows which further depends on the relationship to muscle attachments of face and finally causing the perforation in the cortical plate. After formation of sinus tract, inflammation at the root apex may persist for a longer time because of the drainage through the sinus and hence, a chronic abscess may remain asymptomatic for longer period of time. ${ }^{5}$

With correct diagnosis and definitive treatment, the tract is expected to disappear in a span of 7 to 14 days. Once the root canal system has been thoroughly cleansed, the sinus heals rapidly without the need of any adjunctive therapy such as systemic antibiotics.

Radiographs are of utmost importance to rule out other possible pathologies and establish a final diagnosis coinciding with the patient's signs and symptoms. The newer 3 dimensional CBCT imaging system, has been successfully used in the field of endodontics in various aspects like early detection of apical periodontitis and bony lesions, identification of any aberrant anatomy of tooth and evaluation of root canal preparation and obturation. ${ }^{6}$ In this case, it helped to confirm the size and extent of bone destruction around the apices of tooth \#47.

Differential diagnosis of a cutaneous draining sinus tract may be suppurative apical periodontitis, congenital fistula, salivary gland fistula, osteomyelitis, infected cyst, deep mycotic infection and pyogenic granuloma to prevent any misdiagnosis. ${ }^{7}$

\section{CONCLUSIONS}

Timely and correct diagnosis with a definitive treatment is the key to treat sinus tracts. Successful management of odontogenic extraoral sinus tracts having pulpal pathology depends on the proper diagnosis along with removal of aetiological factors by adequate bio- and chemo-mechanical preparation and a three-dimensional obturation. A thorough evaluation, both clinical and radio graphical, as well as patient's history plays a major role in the accurate diagnosis and treatment of the tooth.

Financial or other competing interests: None.

Disclosure forms provided by the authors are available with the full text of this article at jemds.com.

\section{REFERENCES}

[1] Kumar SK, Subbiya A, Vivekanandhan P, et al. Management of an endodontic infection with an extra oral sinus tract in a single visit: a case report. J Clin Diagn Res 2013;7(6):1247-9. 
[2] Dincol ME, Yilmaz B, Ersev H, et al. Treatment of extra oral cutaneous sinus tracts with non-surgical endodontic intervention: report of six cases. J Istanb Univ Fac Dent 2015;49(2):35-40.

[3] Cohenca N, Karni S, Rotstein I. Extraoral sinus tract misdiagnosed as an endodontic lesion. Journal of Endodontics 2003;29(12):841-3.

[4] Giménez-García R, Martinez-Vera F, Fuentes-Vera L. Cutaneous sinus tracts of odontogenic origin: two case reports. J Am Board Fam Med 2015;28(6):838-40.
[5] Uppal A, Thakur S, Chauhan D, et al. Extraoral cutaneous sinus tracts of dental origin: a report of two pedodontic cases. International Dental \& Medical Journal of Advanced Research 2017;2015(3):1-4.

[6] Tian J, Liang G, Qi W, et al. Odontogenic cutaneous sinus tract associated with a mandibular second molar having a rare distolingual root: a case report. Head Face Med 2015;11:13.

[7] Padhye L, Jagan IN, Unnikrishnan P, et al. Management of extra oral sinus via nonsurgical root canal treatment. Indian J Oral Health Res 2018;4:66-9. 